Proyecciones Journal of Mathematics

Vol. 32, No 4, pp. 347-357, December 2013.

Universidad Católica del Norte

Antofagasta - Chile

\title{
On the generating matrices of the $k$-Fibonacci numbers
}

\author{
Sergio Falcon \\ Universidad de las Palmas, España \\ Received : February 2013. Accepted : September 2013
}

\begin{abstract}
In this paper we define some tridiagonal matrices depending of a parameter from which we will find the $k$-Fibonacci numbers. And from the cofactor matrix of one of these matrices we will prove some formulas for the $k$-Fibonacci numbers differently to the traditional form. Finally, we will study the eigenvalues of these tridiagonal matrices.
\end{abstract}

Keyword : k-Fibonacci numbers, Cofactor matrix, Eigenvalues.

AMS : 11B39, 34L16. 


\section{Introduction}

The generalization of the Fibonacci sequence has been treated by some authors as e.g. Hoggat V.E. [6] and Horadam A.F. [7].

One of these generalizations has been found by Falcon S. and Plaza A. to study the method of triangulation 4TLE [1] and that we define below.

We define the $k$-Fibonacci numbers $[1,2,3]$ by mean of the recurrence relation $F_{k, n+1}=k F_{k, n}+F_{k, n-1}$ for $n \geq 1$ with the initial conditions $F_{k, 0}=0$ and $F_{k, 1}=1$.

The recurrence equation of this formula is $r^{2}-k \cdot r-1=0$ whose solutions are $\sigma_{1}=\frac{k+\sqrt{k^{2}+4}}{2}$ and $\sigma_{2}=\frac{k-\sqrt{k^{2}+4}}{2}$.

The Binet formula for these numbers is $F_{k, n}=\frac{\sigma_{1}^{n}-\sigma_{2}^{n}}{\sigma_{1}-\sigma_{2}}$

From the definition of the $k$-Fibonacci numbers, the first of them are presented in Table ??.

\section{First $k$-Fibonacci numbers}

$$
\begin{aligned}
& \mathrm{F}_{k, 0}=0 \\
& \mathrm{~F}_{k, 1}=1 \\
& \mathrm{~F}_{k, 2}=k \\
& \mathrm{~F}_{k, 3}=k^{2}+1 \\
& \mathrm{~F}_{k, 4}=k^{3}+2 k \\
& \mathrm{~F}_{k, 5}=k^{4}+3 k^{2}+1 \\
& \mathrm{~F}_{k, 6}=k^{5}+4 k^{3}+3 k
\end{aligned}
$$

For $k=1$, the classical Fibonacci sequence $\{0,1,1,2,3,5,8, \ldots\}$ is obtained and for $k=2$ it is the Pell sequence $\{0,1,2,5,12,29, \ldots\}$

\section{Tridiagonal matrices and the $k$-Fibonacci numbers}

In this section we extend the matrices defined in [4] and applied them to the $k$-Fibonacci numbers in order to prove some formulas differently to the traditional form. 


\subsection{The determinant of a special kind of tridiagonal matrices}

Let us consider the n-by-n tridiagonal matrices $M_{n}$,

$$
M_{n}=\left(\begin{array}{ccccccccc}
a & b & & & & & & & \\
c & d & e & & & & & & \\
& c & d & e & & & & & \\
& & c & d & e & & & & \\
& & & & \ddots & \ddots & \ddots & & \\
& & & & & & c & d & e \\
& & & & & & & c & d
\end{array}\right)
$$

Solving the sequence of determinants, we find

$$
\begin{aligned}
\left|M_{1}\right| & =a \\
\left|M_{2}\right| & =d \cdot\left|M_{1}\right|-b \dot{c} \\
\left|M_{3}\right| & =d \cdot\left|M_{2}\right|-c \dot{e} \cdot\left|M_{1}\right| \\
\left|M_{4}\right| & =d \cdot\left|M_{3}\right|-c \dot{e} \cdot\left|M_{2}\right| \\
\ldots &
\end{aligned}
$$

And, in general,

$$
\left|M_{n+1}\right|=d \cdot\left|M_{n}\right|-c \dot{e} \cdot\left|M_{n-1}\right|
$$

\subsection{Some tridiagonal matrices and the $k$-Fibonacci numbers}

- If $a=d=k, b=e=1$, and $c=-1$, the matrices $M_{n}$ are transformed in the tridiagonal matrices

$$
H_{n}(k)=\left(\begin{array}{cccccccc}
k & 1 & & & & & & \\
-1 & k & 1 & & & & & \\
& -1 & k & 1 & & & & \\
& & & \ddots & \ddots & \ddots & & \\
& & & & & -1 & k & 1 \\
& & & & & & -1 & k
\end{array}\right)
$$

In this case, and taking into account Table ??, the above formulas are transformed in

$$
\begin{aligned}
\left|H_{1}(k)\right| & =k=F_{k, 2} \\
\left|H_{2}(k)\right| & =k \cdot k-1(-1)=k^{2}+1=F_{k, 3} \\
\left|H_{3}(k)\right| & =k\left(k^{2}+1\right)-(-1) 1 k=k^{3}+2 k=F_{k, 4}
\end{aligned}
$$

and Formula (2.1) is $\left|H_{n}(k)\right|=F_{k, n+1}$ for $n \geq 1$. 
The $k$-Fibonacci numbers can also be obtained from the symmetric tridiagonal matrices

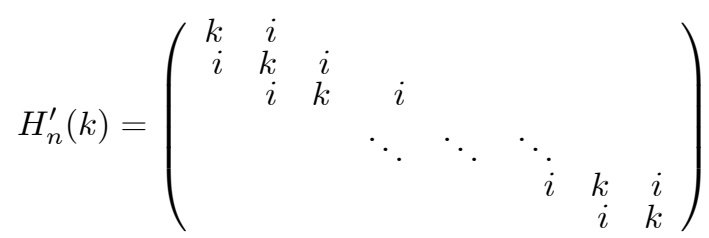

where $i$ is the imaginary unit, i.e. $i^{2}=-1$.

- If $a=k^{2}+1, b=c=e=1, d=k^{2}+2$, the tridiagonal matrices obtained are

$$
O_{n}(k)=\left(\begin{array}{cccccccc}
k^{2}+1 & 1 & & & & & & \\
1 & k^{2}+2 & 1 & & & & & \\
& 1 & k^{2}+2 & 1 & & & & \\
& & & \ddots & \ddots & \ddots & & \\
& & & & & 1 & k^{2}+2 & 1 \\
& & & & & & 1 & k^{2}+2
\end{array}\right)
$$

In this case, it is $\left|O_{n}(k)\right|=F_{k, 2 n+1}$ for $n \geq 1$. So, with $\left|O_{0}(k)\right|=F_{k, 1}=$ 1 , the sequence of these determinants is the sequence of odd $k$-Fibonacci numbers $\left\{1, k^{2}+1, k^{4}+3 k^{2}+1\right\}$.

- Finally, if $a=k, b=0, c=1, d=k^{2}+2$, and $e=1$, for $n \geq 1$ we obtain the even $k$-Fibonacci numbers from the determinant of the matrices

$$
E_{n}(k)=\left(\begin{array}{cccccccc}
k & 0 & & & & & & \\
1 & k^{2}+2 & 1 & & & & & \\
& 1 & k^{2}+2 & 1 & & & & \\
& & & \ddots & \ddots & \ddots & & 1 \\
& & & & & 1 & k^{2}+2 & 1 \\
& & & & & & k^{2}+2
\end{array}\right)
$$

because $\left|E_{n}(k)\right|=F_{k, 2 n}$ for $n \geq 1$ with $\left|E_{0}(k)\right|=F_{k, 0}=0$.

\section{Cofactor matrices of the generating matrices of the $k$ - Fibonacci numbers}

The following definitions are well-known: [9]

If $A$ is a square matrix, then the minor of its entry $a_{i j}$, also known as the $(i, j)$ minor of $A$, is denoted by $M_{i j}$ and is defined to be the determinant of the submatrix obtained by removing from $A$ its $i-t h$ row and $j-t h$ column.

It follows $C_{i j}=(-1)^{i+j} M_{i j}$ and $C_{i j}$ called the cofactor of $a_{i j}$, also referred to as the $(i, j)$ cofactor of $A$. 
Define the cofactor matrix of $A$, as the $n \times n$ matrix $C$ whose $(i, j)$ entry is the $(i, j)$ cofactor of $A$.

Finally, the inverse matrix of $A$ is $A^{-1}=\frac{1}{|A|} C^{T}$, where $|A|$ is the determinant of the matrix $A$ (assuming non zero) and $C^{T}$ is the transpose of the cofactor matrix $C$ or adjugate matrix of $A$.

On the other hand, let us consider the n-by-n nonsingular tridiagonal matrix

$$
T=\left(\begin{array}{ccccc}
a_{1} & b_{1} & & & \\
c_{1} & a_{2} & b_{2} & & \\
& c_{2} & \ddots & \ddots & \\
& & \ddots & \ddots & b_{n-1} \\
& & & c_{n-1} & a_{n}
\end{array}\right)
$$

In [8], Usmani gave an elegant and concise formula for the inverse of the tridiagonal matrix $T^{-1}=\left(t_{i, j}\right)$ :

$$
t_{i, j}=\left\{\begin{array}{l}
(-1)^{i+j} \frac{1}{\theta_{n}} b_{i} \cdots b_{j-1} \theta_{i-1} \phi_{j+1} \quad \text { if } \quad i \leq j \\
(-1)^{i+j} \frac{1}{\theta_{n}} c_{j} \cdots c_{i-1} \theta_{j-1} \phi_{i+1} \quad \text { if } \quad i>j
\end{array}\right.
$$

where

- $\theta_{i}$ verify the recurrence relation $\theta_{i}=a_{i} \theta_{i-1}-b_{i-1} c_{i-1} \theta_{i-2}$ for $i=2, \ldots n$

with the initial conditions $\theta_{0}=1$ and $\theta_{1}=a_{1}$.

Formula (2.1) is one special case of this one.

- $\phi_{i}$ verify the recurrence relation

$\phi_{i}=a_{i} \phi_{i+1}-b_{i} c_{i} \phi_{i+2}$ for $i=n-1, \ldots 1$

with the initial conditions $\phi_{n+1}=1$ and $\phi_{n}=a_{n}$

Observe that $\theta_{n}=\operatorname{det}(T)$.

\subsection{Cofactor matrix of $H_{n}(k)$}

For the matrix $H_{n}(k)$, it is $a_{i}=k, b_{i}=1, c_{i}=-1, \theta_{i}=F_{k, i+1}$ and $\phi_{j}=F_{k, n-j+2}$. Consequently,

$$
\left(\left(H_{n}(k)\right)^{-1}\right)_{i, j}= \begin{cases}(-1)^{i+j} \frac{1}{F_{k, n+1}} F_{k, i} \cdot F_{k, n-j+1} & \text { if } i \leq j \\ \frac{1}{F_{k, n+1}} F_{k, j} \cdot F_{k, n-i+1} & \text { if } \quad i>j\end{cases}
$$


We will work with the cofactor matrix whose entries are

$$
c_{i, j}\left(H_{n}(k)\right)=\left\{\begin{array}{cc}
(-1)^{i+j} F_{k, j} F_{k, n-i+1} & \text { if } i \geq j \\
F_{k, i} F_{k, n-j+1} & \text { if } i<j
\end{array}\right.
$$

So, $c_{j, i}\left(H_{n}(k)\right)=(-1)^{i+j} c_{i, j}\left(H_{n}(k)\right)$ if $i>j$.

In this form, the cofactor matrix of $H_{n}(k)$ for $n \geq 2$ is $C_{n-1}(k)=$

$$
\left(\begin{array}{ccccccc}
F_{k, n} & F_{k, n-1} & F_{k, n-2} & F_{k, n-3} & \cdots & F_{k, 2} & F_{k, 1} \\
-F_{k, n-1} & F_{k, 2} F_{k, n-1} & F_{k, 2} F_{k, n-2} & F_{k, 2} F_{k, n-3} & \cdots & F_{k, 2} F_{k, 2} & F_{k, 2} \\
F_{k, n-2} & -F_{k, 2} F_{k, n-2} & F_{k, 3} F_{k, n-2} & F_{k, 3} F_{k, n-3} & \cdots & F_{k, 3} F_{k, 2} & F_{k, 3} \\
-F_{k, n-3} & F_{k, 2} F_{k, n-3} & -F_{k, 3} F_{k, n-3} & F_{k, 4} F_{k, n-3} & \cdots & F_{k, 4} F_{k, 2} & F_{k, 4} \\
\ddot{F_{k, 2}} & -F_{k, 2} F_{k, 2} & F_{k, 3} \dot{F}_{k, 2} & -F_{k, 4} F_{k, 2} & \cdots & F_{k, n-1} F_{k, 2} & F_{k, n-1} \\
-F_{k, 1} & F_{k, 2} & -F_{k, 3} & F_{k, 4} & \cdots & -F_{k, n-1} & F_{k, n}
\end{array}\right)
$$

On the other hand, taking into account the inverse matrix $A^{-1}=\frac{1}{|A|} A d j(A)$, it is easy to prove $|\operatorname{Adj}(A)|=|A|^{n-1}$.

So, $\left|C_{n-1}(k)\right|=F_{k, n+1}^{n-1}$.

In this form, for $n=2,3,4, \ldots$, it is

$$
\begin{aligned}
& C_{1}(k)=\left|\begin{array}{cc}
F_{k, 2} & F_{k, 1} \\
-F_{k, 1} & F_{k, 2}
\end{array}\right|=F_{k, 3} \rightarrow F_{k, 2}^{2}+F_{k, 1}^{2}=F_{k, 3} \\
& C_{2}(k)=\left|\begin{array}{ccc}
F_{k, 3} & F_{k, 2} & F_{k, 1} \\
-F_{k, 2} & F_{k, 2} F_{k, 2} & F_{k, 2} \\
F_{k, 1} & -F_{k, 2} & F_{k, 3}
\end{array}\right|=F_{k, 4}^{2} \\
& \rightarrow F_{k, 2}^{2}\left(F_{k, 3}^{2}+2 F_{k, 3}+1\right)=F_{k, 4}^{2} \rightarrow\left(\frac{F_{k, 3}-F_{k, 1}}{k}\right)^{2}\left(F_{k, 3}+F_{k, 1}\right)^{2}=F_{k, 4}^{2} \\
& \rightarrow F_{k, 3}^{2}-F_{k, 1}^{2}=k F_{k, 4} \\
& C_{3}(k)=\left|\begin{array}{cccc}
F_{k, 4} & F_{k, 3} & F_{k, 2} & F_{k, 1} \\
-F_{k, 3} & F_{k, 2} F_{k, 3} & F_{k, 2} F_{k, 2} & F_{k, 2} \\
F_{k, 2} & -F_{k, 2} F_{k, 2} & F_{k, 3} F_{k, 2} & F_{k, 3} \\
-F_{k, 1} & F_{k, 2} & -F_{k, 3} & F_{k, 4}
\end{array}\right|=F_{k, 5}^{3} \\
& \rightarrow\left(F_{k, 2}^{2}+F_{k, 3}^{2}\right) F_{k, 5}^{2}=F_{k, 5}^{3} \rightarrow F_{k, 2}^{2}+F_{k, 3}^{2}=F_{k, 5} \\
& C_{4}(k)=F_{k, 6}^{4} \rightarrow F_{k, 3}^{2}\left(F_{k, 2}+F_{k, 4}\right)^{2} F_{k, 6}^{2}=F_{k, 6}^{4} \\
& \rightarrow\left(\frac{F_{k, 4}-F_{k, 2}}{k}\right)^{2}\left(F_{k, 4}+F_{k, 2}\right)^{2}=F_{k, 6}^{2} \rightarrow F_{k, 4}^{2}-F_{k, 2}^{2}=k F_{k, 6}
\end{aligned}
$$

Generalizing these results, and taking into account $F_{k, n}=\frac{F_{k, n+1}-F_{k, n-1}}{k}$, we find the following two formulas for the $k$-Fibonacci numbers according to that $n$ is odd or even [2]: $F_{k, n+1}^{2}+F_{k, n}^{2}=F_{k, 2 n+1}$ and $F_{k, n+1}^{2}-F_{k, n-1}^{2}=k \cdot F_{k, 2 n}$ 


\subsection{Cofactor matrix of $O_{n}(k)$}

To apply formula (3.2) to the matrices $O_{n}(k)$, we must take into account that

$$
\begin{aligned}
a_{1} & =k^{2}+1 \\
a_{i} & =k^{2}+2, i \geq 2 \\
b_{i} & =c_{i}=1, i \geq 1 \\
\theta_{i} & =F_{k, 2 i-1}, i \geq 1 \\
\phi_{j} & =\frac{1}{k} F_{k, 2(n-j+2)}, j \geq 1
\end{aligned}
$$

and consequently the cofactor of the $(i, j)$ entry of these matrices is

$$
\begin{gathered}
c_{i, j}\left(O_{n}(k)\right)=(-1)^{i+j} \frac{1}{k} F_{k, 2 j-1} F_{k, 2(n-i+1)} \text { if } i \geq j \\
c_{j, i}\left(O_{n}(k)\right)=c_{i, j}\left(O_{n}(k)\right) \text { for } j>i
\end{gathered}
$$

\subsection{Cofactor matrix of $E_{n}(k)$}

For the matrices $E_{n}(k)$ it is

$$
\begin{aligned}
a_{1} & =k=F_{k, 2} \\
a_{i} & =k^{2}+2, i \geq 2 \\
b_{1} & =0 \\
b_{i+1} & =c_{i}=1, i \geq 1 \\
\theta_{i} & =F_{k, 2(i+1)}, i \geq 1 \\
\phi_{j} & =\frac{1}{k} F_{k, 2(n-j+2)}, j \geq 1
\end{aligned}
$$

and consequently the cofactor of the $(i, j)$ entry of these matrices is

$$
\begin{gathered}
c_{1, j}\left(E_{n}(k)\right)=(-1)^{j+1} \frac{1}{k} F_{k, 2(n-j+1)} \\
c_{i, j}\left(E_{n}(k)\right)=(-1)^{i+j} \frac{1}{k} F_{k, 2 j} F_{k, 2(n-i+1)}, \quad \text { if } \quad i \geq j, \quad i>1 \\
\mathrm{c}_{j, i}\left(E_{n}(k)\right)=c_{i, j}\left(E_{n}(k)\right), \quad \text { if } j>i>1
\end{gathered}
$$

\section{Eigenvalues}

This section is dedicated to the study of the eigenvalues of the matrices $H_{n}(k)$, $O_{n}(k)$ and $E_{n}(k)$. 


\subsection{Eigenvalues of the matrices $H_{n}(k)$}

The matrix (3.1) has entries in the diagonals $a_{1}, \ldots, a_{n}, b_{1}, \ldots, b_{n-1}, c_{1}, \ldots, c_{n-1}$.

It is well-known the eigenvalues of the matrix (3.1) are

$\lambda_{r}=a+2 \sqrt{b \cdot c} \cos \left(\frac{r \pi}{n+1}\right)$ for $r=1,2, \ldots, n$.

Consequently, the eigenvalues of the matrix $H_{n}(k)$ where $a=k, b=1, c=-1$, are $\lambda_{r}=k+2 i \cos \left(\frac{r \pi}{n+1}\right)$

If $n$ is odd, then the matrix $H_{n}(k)$ has one unique real eigenvalue corresponding to $r=\frac{n+1}{2}$.

If $n$ is even, no one eigenvalue is real.

So, the sequence of spectra of the tridiagonal matrices $H_{n}(k)$ for $n=1,2, \ldots$. is

$$
\begin{aligned}
& \Sigma_{1}=\{k\} \\
& \Sigma_{2}=\{k \pm i\} \\
& \Sigma_{3}=\{k, k \pm i \sqrt{2}\} \\
& \Sigma_{4}=\left\{k \pm \frac{1+\sqrt{5}}{2} i, k \pm \frac{1-\sqrt{5}}{2} i\right\}=\left\{k \pm \phi i, k \pm(-\phi)^{-1} i\right\} \\
& \Sigma_{5}=\{k, k \pm i, k \pm i \sqrt{3}\} \\
& \ldots
\end{aligned}
$$

where $\phi=\frac{1+\sqrt{5}}{2}$ is the Golden Ratio.

All the roots $\lambda_{r}$ lie on the segment $\Re\left(\lambda_{j}\right)=k,-2<\Im\left(\lambda_{j}\right)<2$.

It is verified $\sum_{j=1}^{n} \lambda_{j}=n k$ and $\prod_{j=1}^{n} \lambda_{j}=F_{k, n+1}$.

Moreover, taking into account the product of all eigenvalues is the determinant of the matrix $H_{n}(k)$ and as $\left|H_{n}(k)\right|=F_{k, n+1}$, it is verified that

$$
F_{k, n+1}=\prod_{j=1}^{n}\left(k+2 i \cos \left(\frac{\pi j}{n+1}\right)\right)
$$

\subsection{Eigenvalues of the matrices $O_{n}(k)$}

Matrices $O_{n}(k)$ are symmetric, so all its eigenvalues are real. 


\subsubsection{Theorem}

If $\lambda_{i}$ is an eigenvalue of the matrix $O_{n}(k)$ for a fixed value $k$, then $\lambda_{i}+2 k+1$ is eigenvalue of the matrix $O_{n}(k+1)$.

Proof. If $\lambda_{i}$ is an eigenvalue of the matrix $O_{n}(k)$, then it is

$$
\begin{aligned}
& \left|O_{n}(k)-\lambda_{i} I_{n}\right|=\left|\begin{array}{cccccc}
k^{2}+1-\lambda_{i} & 1 & & & & \\
1 & k^{2}+2-\lambda_{i} & 1 & & \\
& 1 & k^{2}+2-\lambda_{i} & 1 & \\
& & & \ddots & \ddots & \ddots
\end{array}\right|= \\
& \begin{array}{ccccc}
(k+1)^{2}+1-\left(\lambda_{i}+2 k+1\right) & 1 & & & \\
& (k+1)^{2}+2-\left(\lambda_{i}+2 k+1\right) & & & \\
& & (k+1)^{2}+2-\left(\lambda_{i}+2 k+1\right) & 1 & \\
& & \ddots & \ddots
\end{array} \mid= \\
& \left|O_{n}(k+1)-\left(\lambda_{i}+2 k+1\right) I_{n}\right|
\end{aligned}
$$

Consequently, only it is necessary to find the eigenvalues of the matrix $O_{n}(1)$ for $n=2,3, \ldots$ and then, if $\lambda_{j}$ is an eigenvalue of $O_{n}(1)$, then

$$
\lambda_{j}^{\prime}=\lambda_{j}+k^{2}-1
$$

is an eigenvalue of the matrix $O_{n}(k)$.

Now we will study the spectra of these matrices.

First, we present the spectra of these matrices for $k=1$ and $n=2,3,4,5,6,7,8$ obtained with the help of MATHEMATICA:

$\Sigma_{2}=\{1.381966,3.618034\}$

$\Sigma_{3}=\{1.198062,2.554958,4.246980\}$

$\Sigma_{4}=\{1.120615,2.000000,3.347296,4.532089\}$

$\Sigma_{5}=\{1.081014,1.690279,2.715370,3.830830,4.682507\}$

$\Sigma_{6}=\{1.058116,1.502979,2.290790,3.241073,4.136129,4.770912\}$

$\Sigma_{7}=\{1.043705,1.381966,2.000000,2.790943,3.618034,4.338261,4.827091\}$

$\Sigma_{8}=\{1.034054,1.299566,1.794731,2.452674,3.184537,3.891477,4.478018,4.864944\}$

Evidently,

$$
\sum \lambda_{j}=3 n-1
$$

and

$$
\prod \lambda_{j}=F_{2 n+1}
$$

Below are the minimum and maximum eigenvalues of these spectra:

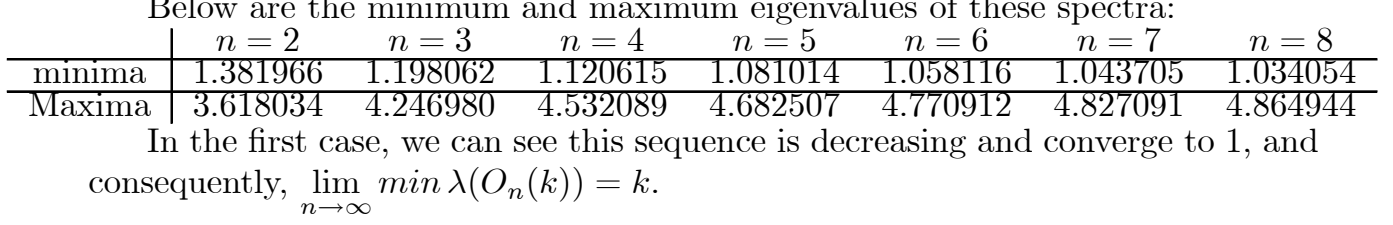


In the same form, the sequence of Maxima is increasing and converge to 5 , so we can say $\lim _{n \rightarrow \infty} \operatorname{Max} \lambda\left(O_{n}(k)\right)=k^{2}+4$.

Finally, if $k \neq 1$, then, taking into account Formula (4.1), the formulas (4.2) and (4.3) are transformed into

$$
\begin{aligned}
\sum_{j=1}^{n} \lambda_{j}(k) & =\sum\left(\lambda_{i}(1)+k^{2}-1\right)=n k^{2}+2 n-1 \\
\prod_{j=1}^{n} \lambda_{j}(k) & =F_{k, 2 n+1}
\end{aligned}
$$

\subsection{Eigenvalues of the matrices $E_{n}(k)$}

Finally, we say a matrix is positive if all the entries are real and nonnegative. If a matrix is tridiagonal and positive, then all the eigenvalues are real [5]. So, taking into account matrix $E_{n}(k)$ is tridiagonal and positive, all its eigenvalues are real.

Following the same process that for the matrices $O_{n}(k)$, we can prove that the first eigenvalue is $k$ and the others verify $\lambda_{i}(k)=\lambda_{i}(1)+k^{2}-1$.

Moreover, $\sum_{j=1}^{n} \lambda_{j}(k)=(n-1)\left(k^{2}+2\right)+k$ and $\prod_{j=1}^{n} \lambda_{j}(k)=F_{k, 2 n}$

The sequence of spectra of the matrices $E_{n}(1)$ is

$\Sigma_{2}=\{1,3\}$

$\Sigma_{3}=\{1,2,4\}$

$\Sigma_{4}=\{1,1.585786,3,4.414214\}$

$\Sigma_{5}=\{1,1.381966,2.381966,3.618034,4.618034\}$

$\Sigma_{6}=\{1,1.267949,2,3,4,4.732051\}$

$\sigma_{7}=\{1,1.198062,1.753020,2.554958,3.445042,4.246980,4.801938\}$

The sequence of minima eigenvalue converges to 1 (to $k$ in general ) and the sequience of maxima converges to 5 (to $k^{2}+4$ in the general case).

\section{Acknowledgements.}

This work has been supported in part by CICYT Project number MTM200805866-C03-02/MTM from Ministerio de Educación y Ciencia of Spain.

\section{References}

[1] Falcon S. and Plaza A., On the Fibonacci $k$-numbers, Chaos, Solit. \& Fract. 32 (5), pp. 1615-1624, (2007). 
[2] Falcon S. and Plaza A., The $k$-Fibonacci sequence and the Pascal 2-triangle, Chaos, Solit. \& Fract. 33 (1), pp. 38-49, (2007).

[3] Falcon S. and Plaza A., The $k$-Fibonacci hyperbolic functions, Chaos, Solit. \& Fract. 38 (2), pp. 409-420, (2008).

[4] Feng A., Fibonacci identities via determinant of tridiagonal matrix, Applied Mathematics and Computation, 217, pp. 5978-5981, (2011).

[5] Horn R. A. and Johnson C. R., Matrix Analysis, p. 506, Cambridge University Press (1991)

[6] Hoggat V. E. Fibonacci and Lucas numbers, Houghton-Miffin, (1969).

[7] Horadam A. F. A generalized Fibonacci sequence, Mathematics Magazine, 68, pp. 455-459, (1961).

[8] Usmani R., Inversion of a tridiagonal Jacobi matrix, Linear Algebra Appl. 212/213, pp. 413-414, (1994).

[9] Wikipedia, http://en.wikipedia.org/wiki/Cofactor_matrix

\section{Sergio Falcon}

University of Las Palmas de Gran Canaria,

Department of Mathematics,

35017-Las Palmas de Gran Canaria,

España

e-mail : sfalcon@dma.ulpgc.es 